\title{
High precision Cross-correlated imaging in Few-mode fibers
}

Muliar, Olena; Usuga Castaneda, Mario A.; Kristensen, Torben; Alkeskjold, Thomas Tanggaard; Rottwitt, Karsten; Lægsgaard, Jesper

\section{Published in:}

Proceedings of SPIE

Link to article, DOI:

$10.1117 / 12.2250532$

Publication date:

2017

Document Version

Publisher's PDF, also known as Version of record

Link back to DTU Orbit

Citation (APA):

Muliar, O., Usuga Castaneda, M. A., Kristensen, T., Alkeskjold, T. T., Rottwitt, K., \& Lægsgaard, J. (2017). High precision Cross-correlated imaging in Few-mode fibers. In Proceedings of SPIE (Vol. 10130). [101300T] SPIE International Society for Optical Engineering. Proceedings of SPIE - The International Society for Optical Engineering https://doi.org/10.1117/12.2250532

\section{General rights}

Copyright and moral rights for the publications made accessible in the public portal are retained by the authors and/or other copyright owners and it is a condition of accessing publications that users recognise and abide by the legal requirements associated with these rights.

- Users may download and print one copy of any publication from the public portal for the purpose of private study or research.

- You may not further distribute the material or use it for any profit-making activity or commercial gain

- You may freely distribute the URL identifying the publication in the public portal 


\title{
High precision Cross-correlated imaging in Few-mode fibers
}

\author{
Olena Muliar ${ }^{\mathrm{a}}$, Mario A. Usuga Castaneda ${ }^{\mathrm{a}}$, Torben Kristensen ${ }^{\mathrm{b}}$, Thomas Tanggaard \\ Alkeskjold ${ }^{\mathrm{b}}$, Karsten Rottwitt ${ }^{\mathrm{a}}$, and Jesper Lægsgaard ${ }^{\mathrm{a}}$ \\ ${ }^{a}$ DTU Fotonik, Department of Photonics Engineering, Technical University of Denmark, \\ Ørsteds Plads, 2800 Kgs. Lyngby, Denmark \\ bNKT Photonics, Blokken 84, 3460 Birkerød, Denmark
}

\begin{abstract}
The trend of increasing data traffic in conventional communication systems demands utilizing new methods for data transmission, which in combination with traditional techniques, enable overcoming the predicted capacity limit. Mode division multiplexing (MDM), where higher-order modes (HOMs) in a few-mode fiber (FMF) are used as multiple spatial communication channels, comes in this context as a viable approach to enable the optimization of high-capacity links. From this perspective, it becomes highly necessary to possess a diagnostic tool for the precise modal characterization of FMFs. Among existing approaches for modal content analysis, several methods as $S^{2}, C^{2}$ in time and frequency domain are available.

In this contribution we will present an improved time-domain cross-correlated $\left(C^{2}\right)$ imaging technique for the experimental evaluation of modal properties in HOM fibers over a broad range of wavelengths. Our modified setup makes it possible to adjust the time resolution of the system according to the needs of the required fiber measurement. We show that by tuning the spectral shape of the source (SuperK EXTREME filtered by SuperK Select), we enhance the time resolution of the system, which allows us to distinguishing differential time delays between HOMs in the picosecond timescale. Broad wavelength scanning in combination with spectral shaping, allows us to estimate the modal behavior of FMF without prior knowledge of the fiber parameters. We performed our demonstration at wavelengths from $850 \mathrm{~nm}$ to $1100 \mathrm{~nm}$ which can be easily extended to other wavelengths of interest just by replacing components with the appropriate coating. The method presented here aims to serve as flexible diagnostic tool that can be implemented in MDM systems for judicious evaluation of modal dispersion in FMFs.
\end{abstract}

Keywords: mode division multiplexing, few mode fiber, cross-correlated imaging, mode characterization, higherorder mode

\section{INTRODUCTION}

A growing need of the modern information society for data exchange has resulted in overload of current communication systems leading to the predicted capacity limit of the modern network links. To boost digital data transmission in silica-based optical fiber channels the 5 physical dimensions amplitude, phase, time, wavelength and polarization were exploited. However, restriction of the conventional communication technology does not allow additional increase of link's capacity without further investigation of new approaches and techniques for the data transmission. From this point of view, space-division multiplexing (SDM) and mode-division multiplexing (MDM) appear as promising approaches that can overcome capacity crunch of the standard communication channels bringing breakthrough of the data transmission to Pbit level and are believed to become essential methods for the next generation of the networks.

The idea of SDM as well as MDM appeared in the beginning of $1980 \mathrm{~s},{ }^{1,2}$ but the technology was not mature enough and it took about 3 decades for its implementation to be feasible. ${ }^{3,4}$ The principle of SDM is based on utilization of additional cores in multicore fiber (MCF) as extra channels for the data transmission, while in MDM the separate communication channels are constituted by higher-order modes that propagate in a fewmode (FMF) or a multi-mode fiber (MMF). Up-to-date achievements carried out in the laboratory demonstrate

Further author information: (Send correspondence to O.Muliar)

O.Muliar: E-mail: omul@fotonik.dtu.dk, Telephone: +45 45 256632

Next-Generation Optical Communication: Components, Sub-Systems, and Systems VI,

edited by Guifang Li, Xiang Zhou, Proc. of SPIE Vol. 10130, 101300T · @ 2017 SPIE

CCC code: $0277-786 X / 17 / \$ 18 \cdot$ doi: $10.1117 / 12.2250532$

Proc. of SPIE Vol. 10130 101300T-1 
MDM of 15 spatial modes over $22.8 \mathrm{~km}^{5}$ in graded-index multimode fiber (GI-MMF) and SDM transmission of 3 modes in 12 cores of graded-index multicore few-mode-fiber (GI-MC-FMF) over 527-km. ${ }^{6}$ To successfully fulfill complex requirements of the future SDM and MDM systems the main focus must be on the transmission medium. Carefully designed FMF and MMF that transmit number of spatial modes with low intermodal coupling and dispersion ${ }^{7}$ are emerging as fiber links for the next-generation of optical networks. In this case, it is extremely important to be able to investigate and verify properties of the designed fibers experimentally. Therefore, an additional diagnostic tool for an experimental characterization of the modal content of FMF and MMF in MDM system is highly beneficial.

A few techniques for the modal content evaluation have by now been presented. Among them are spatially and spectrally resolved imaging $\left(S^{2}\right),{ }^{8}$ cross-correlated imaging in the time ${ }^{9}\left(C^{2}\right)$ and the frequency domain ${ }^{9}\left(f C^{2}\right)$ and matched white-light interferometry (MWI). ${ }^{10}$ All of them are based on the idea of the spatial interference between modes propagating in the same fiber $\left(S^{2}\right)$ or radiated from the sample and the reference fibers, as in the case of Mach-Zehnder interferometer for $C^{2}, f C^{2}$ and WLI. As a result, information about the modal content of the fiber, such as amount of propagating modes, their relative power, intensity distributions and differential time delays can be found.

In this contribution we are going to present an improved cross-correlated $\left(C^{2}\right)$ imaging technique in the time domain with an additional degree of flexibility performing measurements within a broad range of wavelengths and exhibiting overall picture of the modal behavior at deliberately chosen wavelengths. Moreover, due to the modified configuration of the setup we are capable to adjust the temporal resolution of the system in desired way and choose optimal conditions for the fiber content assessment depending on the requirement of the fiber design. The optimized $C^{2}$ imaging technique is appealing also in the context of its implementation as a diagnostic tool in MDM scenario. Demonstration of the method is performed for the common step-index fiber SMF28 at a wavelength range from $870 \mathrm{~nm}$ to $1100 \mathrm{~nm}$, where the fiber under test (FUT) is considered as FMF. Properties of the modes and sensitivity of the setup to the different width and shape of the input spectrum of the light source at different wavelengths are investigated.

\section{RESULTS}

\subsection{Description of the setup for the improved cross-correlated imaging}

In the Figure 1 is presented a scheme of the improved setup for a cross-correlated imaging method. The setup is based on Mach-Zehnder interferometer. As a light source in the optimized $C^{2}$ setup a broadband supercontinuum laser (SuperK EXTREME, NKT Photonics) that transmits light in a region from 400nm to $2400 \mathrm{~nm}$ was used. The incoming spectrum was filter by a multi-line tunable filtering box (SuperSELECT, NKT Photonics) with a spectral bandwidth of $7.23 \mathrm{~nm}$ for one channel. The central filtering wavelength can be chosen along the near infrared region from $800 \mathrm{~nm}$ to $1400 \mathrm{~nm}$. One of the intrinsic features of SuperSELECT is the opportunity to tune simultaneously up to 8 channels. The wavelength of each channel as well as the power can be adjusted separately. An example of the output spectrum of the light source filtered by SuperSELECT with only one radiated channel at the central filtering wavelength of $1050 \mathrm{~nm}$ is depicted as an inset in Figure 3 and has a characteristic sinc-shape with a side-lobes suppression of $-10 \mathrm{~dB}$, when the beam power is $30 \%$ of the maximal power.

To evenly couple light into both arms of the interferometer a PM 50/50 fiber coupler was used. The FUT was a SMF28 fiber that was balanced by a 980HP fiber in the reference arm. Lengths of the FUT and the reference fiber are $1 \mathrm{~m}$ each. The FUT has a cutoff wavelength around 1260nm. According to calculations within the wavelength range of our interest two modes propagate in the FUT: the fundamental mode (FM) and the first HOM. Calculated effective refractive indices for the propagating LP01 and LP11 modes as a function of wavelength are presented in Figure 2.

In both arms linear polarizers control polarization states of the output light. Optical paths of the arms are carefully controlled and matched by a delay line in the reference arm. During the measurements the delay line was programmed to scan with an incremental step of $5 \mu \mathrm{m}$ that corresponds to $16 \mathrm{fs}$ of the time delay along $1 \mathrm{~mm}$ distance. After propagating in the reference fiber and the FUT beams are recombined at the beam-splitter and imaged onto a CCD camera that records interferograms at each step of the displacement of the delay line. The 
duration of the single experimental run usually varies from a few to 20 minutes depending on the number of the incremental steps performed by the delay line. The stack of pictures, acquired by the camera, are analyzed following a procedure described in Ref. ${ }^{9}$ As a result, variation of the intensity distribution of the two interfering beams depending on the time delay is presented as an imaging $C^{2}$ trace that contains information about the modal quantity, differential time delays and dispersion of the modes.

Excitation of the HOMs in the FUT was reached by changing the input coupling condition and introducing an offset between the coupled beam and the fiber core. In this way in the FUT were excited two modes of $40 \%$ and $60 \%$ of modal power corresponding to LP11 and LP01. One of the advantages of $C^{2}$ method is ability to detect modes of equal or close to equal powers. The measured $C^{2}$ trace is shown in Figure 3 (in a blue color). Two modes are present on the trace corresponding to the predicted LP01 and LP11. At the range of wavelengths from $870 \mathrm{~nm}$ and up to its cutoff LP11 propagates with higher group velocity, and therefore during the measurements it comes out as the first detected mode in the $C^{2}$ trace. The red curve represent the theoretically calculated $C^{2}$ trace. As it is seen from Figure 3 experimental and theoretical data are in good agreement. From the positions of the peaks on the trace the differential temporal delay can be derived as a time delay of the HOM with respect to the FM. It was calculated that at the wavelength of $1050 \mathrm{~nm}$ the differential time delay between modes has to be $-1.92 \mathrm{ps} / \mathrm{m}$, while the measured value is $-2 \mathrm{ps} / \mathrm{m}$.

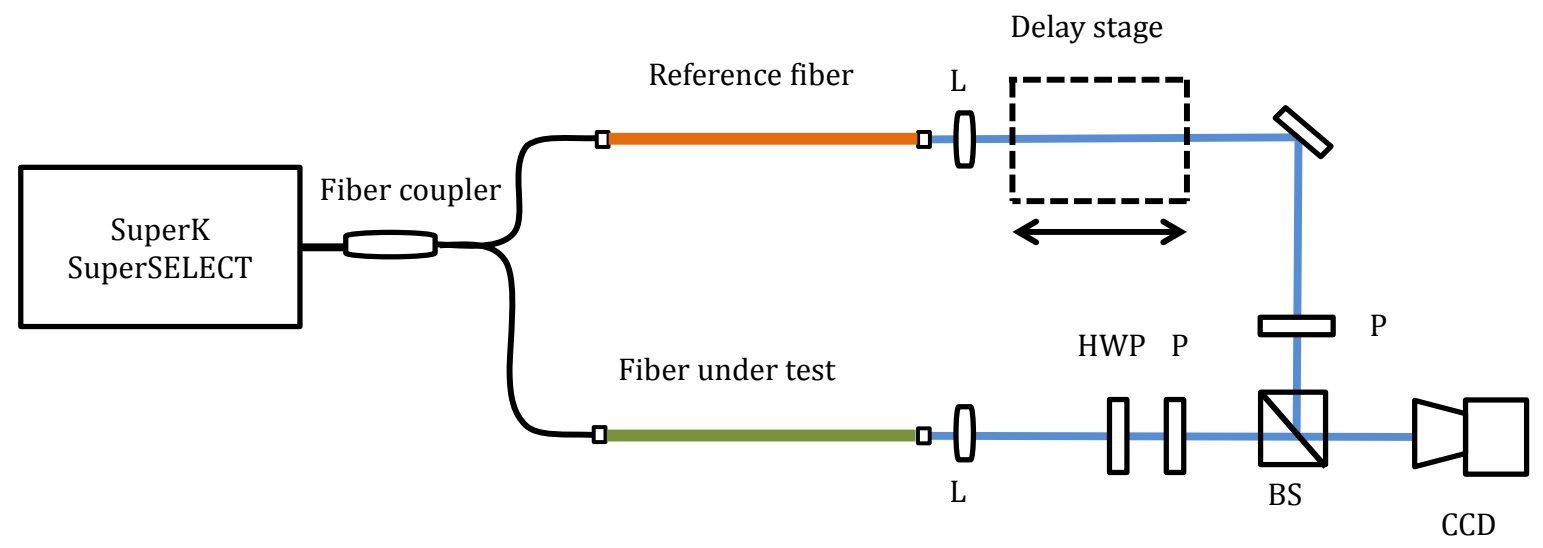

Figure 1. The scheme of the experimental setup for the improved $C^{2}$ measurement. Here, SuperK and Super SELECT are applied in the setup as a light source and a filtering box, PM coupler - polarization maintaining fiber coupler, L lens, P - linear polarizer, BS - beam splitter.

\subsection{Influence of the spectral width and shape on the imaging trace}

One of the powerful features of SuperK Select in combination with SuperK EXTREME is an opportunity to add up to 8 emitting channels at the output increasing in this way the bandpass width and as a consequence the spectral bandwidth of the filtered seed source.The central wavelength and the power of each channel can be separately modified. As an example, $C^{2}$ measurement at $1050 \mathrm{~nm}$ with 1 beam from SuperSELECT was provided with a spectrum depicted in the inset of Figure 3, where the beam power was adjusted to $30 \%$ of the maximal power. Such power level results in the sidelobes suppression up to -10dB in comparison with the main peak. Nevertheless, experimentally it was verified that suppression of the sidelobes lower than -10dB does not affect the appearance of the $C^{2}$ trace. The results of the measurement depicted in Figure 3 confirms that, since the trace still remains smooth and clear. However, the less the shape of the spectrum remains Gaussian, the more affected is the imaging trace. Example of this influence is presented in Figure 4, where the spectrum (depicted in the inset) is combined from 2 merged beams at the central wavelengths of $1040 \mathrm{~nm}$ and $1050 \mathrm{~nm}$ resulting in a split spectrum shape, since the division between the central wavelengths of the beams is larger 


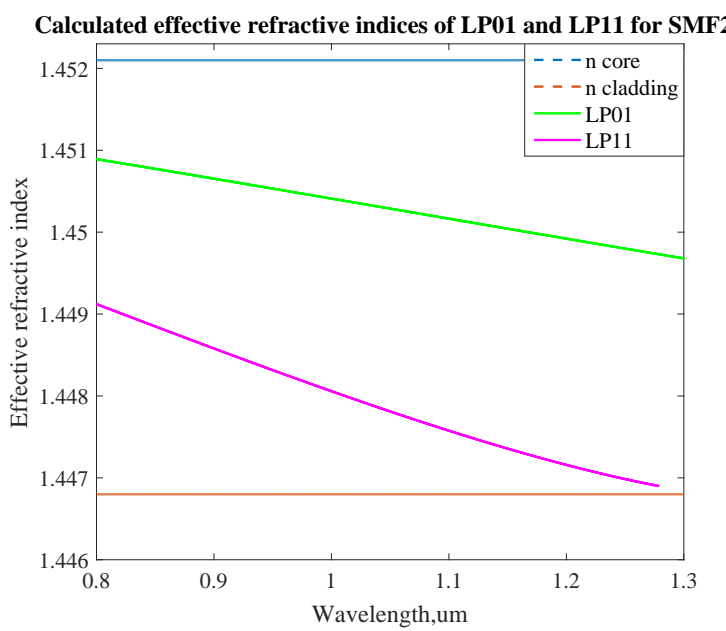

Figure 2. Dependence of the theoretically calculated effective refractive indices of the modes propagating in the FUT (SMF28) as a function of wavelength.

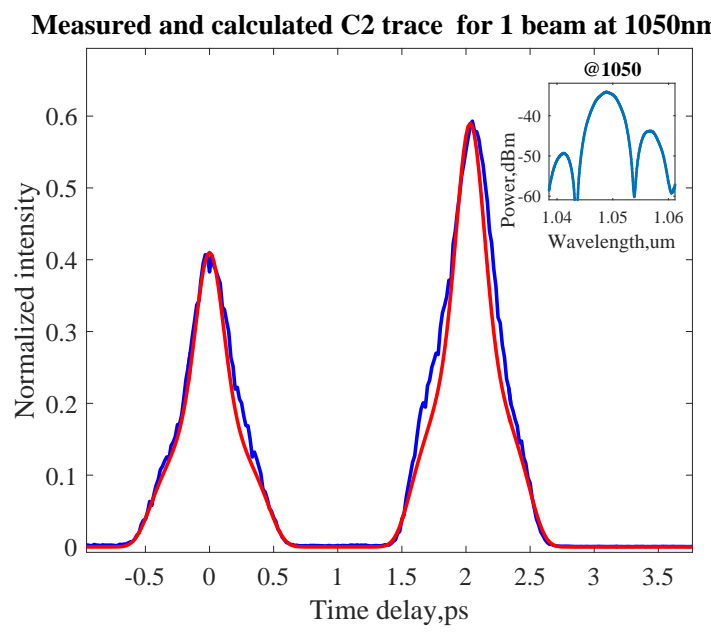

Figure 3. The measured (blue curve) and calculated (red curve) $C^{2}$ imaging trace for one channel from SuperSelect at the central wavelength at $1050 \mathrm{~nm}$. The inset shows the measured spectrum that was implemented in the theoretical calculation and at the measurement.

than the bandwidth of the single channel. Aforesaid disrupted spectral shape results in an appearance of the artificial sidelobes around each interference peak complicating data analysis. Therefore, it is highly important while modulating the spectrum with help of SuperSELECT to take into account dependence of the $C^{2}$ trace appearance on the shape of the spectrum.

To investigate how the temporal resolution of the imaging trace depends on the bandwidth of the seed source we provided $C^{2}$ measurements of SMF28 at the central wavelength of $1050 \mathrm{~nm}$ with the spectra merged from 1,2 , 3,5 and 8 beams presented in Figure 5 . The spectral width was enlarged from $7.23 \mathrm{~nm}$ for 1 beam to $28.25 \mathrm{~nm}$ for a combination of 8 beams that were excited at wavelengths of $1042 \mathrm{~nm}-1045 \mathrm{~nm}-1048 \mathrm{~nm}-1050 \mathrm{~nm}-1052 \mathrm{~nm}-1055 \mathrm{~nm}-$ $1058 \mathrm{~nm}-1061 \mathrm{~nm}$ with power $60 \%-80 \%-100 \%-90 \%-90 \%-70 \%-50 \%-30 \%$ corresponding to each central wavelength. It is worth mentioning that when tuning the spectrum shape for $C^{2}$ measurements an optimal beam combination has to be found to preserve Gaussian-like spectrum shape and maintain the spectral bandwidth wide enough to 


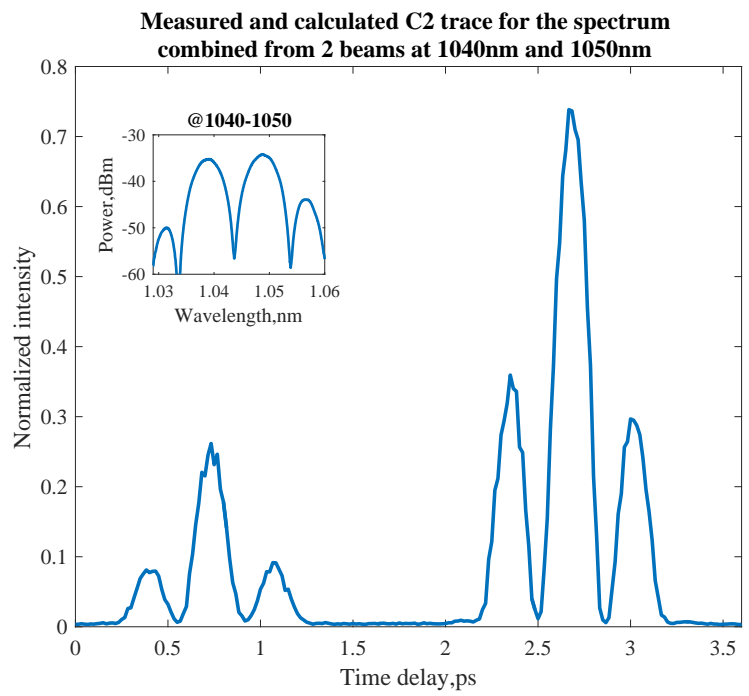

Figure 4. The measured $C^{2}$ imaging trace for 2 turned on channel from SuperSelect with a separation in wavelengths of $10 \mathrm{~nm}$ at the central wavelengths of $1040 \mathrm{~nm}$ and $1050 \mathrm{~nm}$. The inset shows the measured spectrum that was used in the theoretical calculation and at the measurement.

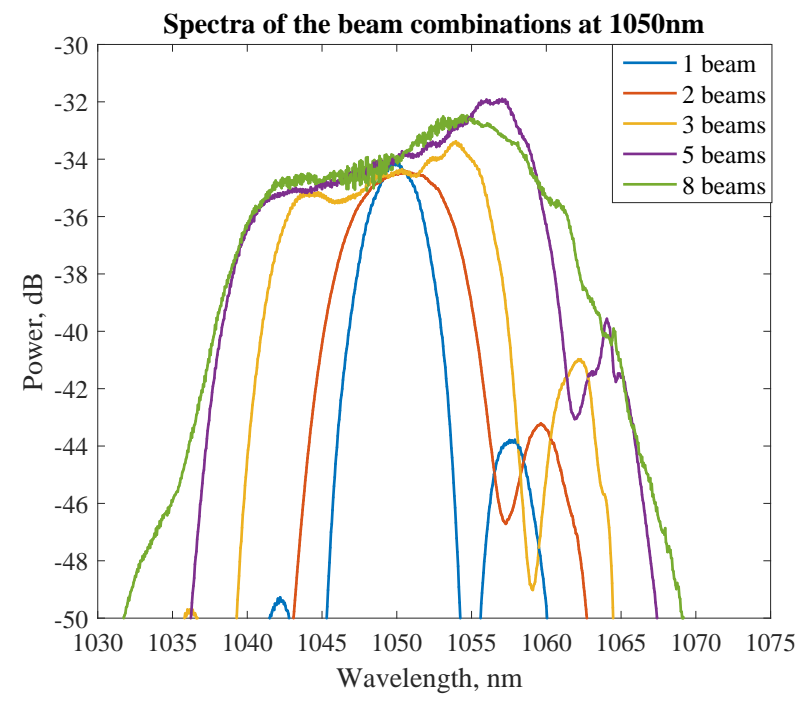

Figure 5. Input spectra merged from 1, 2, 3, 5 and 8 beams.

gain an appropriate temporal resolution for the system. We noticed that separation between beams wider than the FWHM of the 1 irradiated channel $(7.23 \mathrm{~nm})$ affects the trace bringing undesirable artifacts around each peak of interference (Figure 4). However, excitation of the beams with a separation in wavelengths smaller than $2 \mathrm{~nm}$ does not yield desired broadening, but even opposite, it causes interaction between RF signals between channels creating noise fluctuation at the spectrum that can be seen on a spectrum of 8 beams (green line) in Figure 5 . In spite of that the temporal resolution was changed from $0.47 \mathrm{ps}$ for 1 beam to $0.15 \mathrm{ps}$ for 8 beams. Since the width of the interference peaks on the $C^{2}$ trace has a reciprocal dependence on the spectral bandwidth, broader source spectra will lead to more narrow interference peaks and as a consequent to a higher temporal resolution of the $C^{2}$ imaging, when residual dispersion between the arms is matched. 
Figure 6 presents comparison of the $C^{2}$ traces recorded with the aforesaid spectra merged of 1 (blue curve) and 8 beams (red curve). Measured $C^{2}$ trace with wider input spectrum has higher temporal resolution and is only affected to a small degree by the spectral shape, nevertheless it does not disturb detection of the modal content of SMF28 at 1050nm.

\subsection{C2 scan within a broad range of wavelengths}

To investigate behavior of the fiber modal content of SMF28 at different wavelengths the $C^{2}$ measurements were provided at the central wavelength of $870 \mathrm{~nm}, 930 \mathrm{~nm}, 970 \mathrm{~nm}, 1030 \mathrm{~nm}, 1050 \mathrm{~nm}, 1090 \mathrm{~nm}$ and $1100 \mathrm{~nm}$. This selection of wavelengths were chosen due to the operational window of the setup components. In Figure 7 are presented results of the calculated and measured differential time delays derived at each wavelengths of our interest. As it can be seen from Figure 7, experimental and measured values are in good agreement. It is noticeable how the differential time delay between two modes decreases approaching zero value at wavelength close to $870 \mathrm{~nm}$, where the turn around point in the modal group velocities is expected. However, according to the measured data at the wavelength of $870 \mathrm{~nm}$ LP11 mode still remains the first detected mode at the $C^{2}$ trace. We suppose that expected wavelength where LP01 would have higher group velocity is located at wavelength lower than $870 \mathrm{~nm}$.

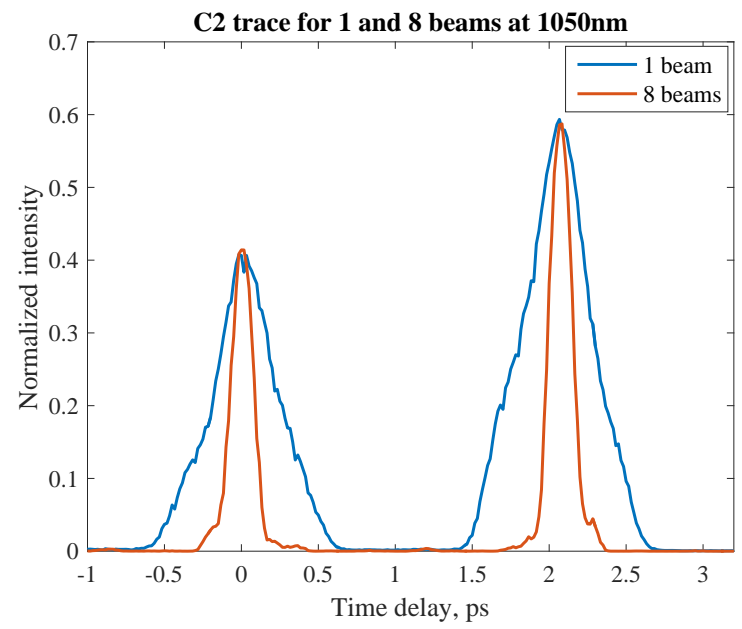

Figure 6. Comparison the $C^{2}$ traces obtained with different input spectra of 1 beam (blue line) and 8 merged beams (red line).

\section{CONCLUSION}

Exploitation of the spatial dimension of the single-mode fiber and its implementation in MDM can help to overcome the capacity limitation of the modern communication links. A technique that can experimentally analyze and verify the theoretically predicted features of the few-mode fibers designed for MDM will be a valuable tool for MDM systems. In the paper, we presented an optimized $C^{2}$ method in the time-domain for the experimental evaluation of the fiber modal content along a broad range of wavelengths from 870 to $1100 \mathrm{~nm}$. Being highly flexible in adjustment of its parameters the setup allows tuning of the spectral width as well as regulation of the spectral shape of the input spectrum enhancing the temporal resolution of the system. Matching the dispersion of the two arms of the interferometer, or in other words, minimizing the influence of the dispersive broadening on the interference peaks' width at the imaging trace, and enlarging the bandwidth of the seed source we reached the temporal resolution of the $C^{2}$ system to be 150fs, which allows us to distinguish differential time delays between HOMs in the picosecond timescale. The modal content of SMF28 was characterized along broad range of wavelengths demonstrating full overview of the modal behavior from $870 \mathrm{~nm}$ to $1100 \mathrm{~nm}$. With the 


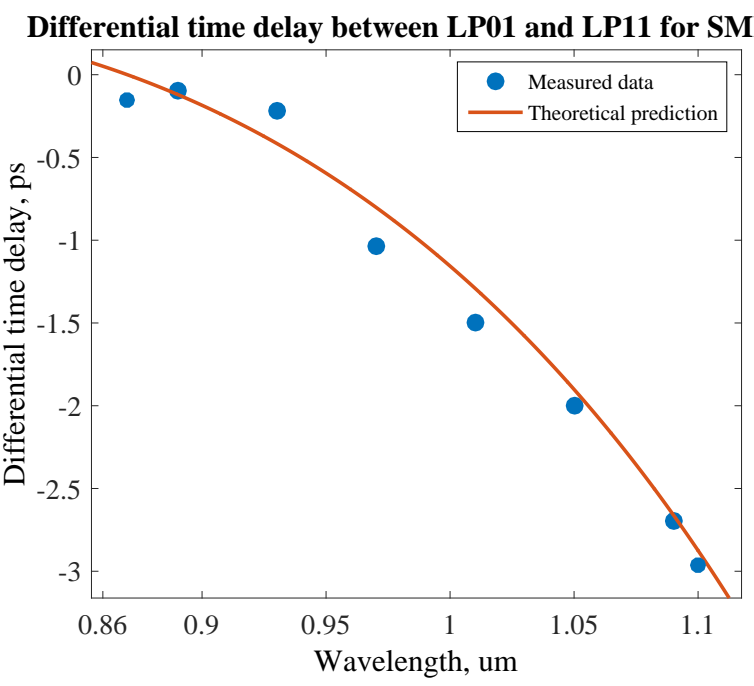

Figure 7. Measured (blue dots) and calculated (red curve) differential time delay between LP01 and LP11 of SMF28.

current setup configuration the optimized $C^{2}$ imaging method is a potential and flexible diagnostic tool with a high degree of variability that can be easy implemented for characterization of fibers with particular design for MDM systems.

\section{REFERENCES}

[1] Iano, S., Sato, T., Sentsui, S., Kuroha, T., and Nishimura, Y., "Multicore optical fiber," Proc. OFC WB1 (1979).

[2] Berdague, S. and Facq, P., "Mode division multiplexing in optical fibers," Appl. Opt. 21, 1950-1955 (1982).

[3] Hanzawa, N., Saitoh, K., Sakamoto, T., Matsui, T., Tomita, S., and Koshiba, M., "Demonstration of mode-division multiplexing transmission over $10 \mathrm{~km}$ two-mode fiber with mode coupler," Proc.OFC OWA4 (2011).

[4] Richardson, D., Fini, J., and Nelson, L., "Space-division multiplexing in optical fibres," Nat. Photonics 7, 354362 (2013).

[5] Fontaine, N., Ryf, R., Chen, H., Benitez, A. V., Lopez, J. E. A., Correa, R. A., Guan, B., Ercan, B., Scott, R. P., Yoo, S., Gruner-Nielsen4, L., Sun, Y., and Lingle, R. J., "30x30 mimo transmission over 15 spatial modes," Proc. OFC Th5C (2015).

[6] Shibahara, K., Mizuno, T., Takara, H., Sano, A., Kawakami, H., Lee, D., Miyamoto, Y., Ono, H., Oguma, M., Abe, Y., Kobayashi, T., Matsui, T., Fukumoto, R., Amma, Y., Hosokawa, T., Matsuo, S., Saito, K., Nasu, H., and Morioka, T., "Dense sdm (12-core x 3-mode) transmission over $527 \mathrm{~km}$ with 33.2-ns modedispersion employing low-complexity parallel mimo frequency-domain equalization," J. Lightw. Technol. 34, 196-204 (2016).

[7] Richardson, D., "New optical fibres for high-capacity optical communications," Phil.Trans.R.Soc.A 374, 116 (2016).

[8] Nicholson, J., Yablon, A. D., Ramachandran, S., and Ghalm, S., "Spatially and spectrally resolved imaging of modal content in large-mode-area fibers," Opt. Express 16 (10), 7233-6243 (2008).

[9] Schimpf, D. N., Barankov, R., and Ramachandran, S., "Cross-correlated (C2) imaging of fiber and waveguide modes," Opt. Express 19 (14), 13008-13019 (2011).

[10] Kong, F., Gu, G., Hawkins, T. W., Parsons, J., Jones, M., Dunn, C., Kalichevsky-Dong, M. T., Palese, S. P., Cheung, E., and Dong, L., "Quantitative mode quality characterization of fibers with extremely large mode areas by matched white-light interferometry," Opt. Express 22 (12), 14657-14665 (2014). 